\title{
PSEUDO-POLYNOMIAL FUNCTIONS OVER FINITE DISTRIBUTIVE LATTICES
}

\author{
MIGUEL COUCEIRO AND TAMÁS WALDHAUSER
}

\begin{abstract}
In this paper we extend the authors previous works by considering a multi-attribute aggregation model based on a composition of a polynomial function over a finite distributive lattice with local utility functions; these are referred to as pseudo-polynomial functions. We present an axiomatization for this class of pseudo-polynomial functions which differs from the previous ones both in flavour and nature, and develop general tools which are then used to obtain all possible such factorizations of a given pseudo-polynomial function.
\end{abstract}

\section{INTRODUCTION AND MOTIVATION}

The Sugeno integral (introduced by Sugeno [17, 18]) remains as one of the most noteworthy aggregation functions, and this is partially due to the fact that it provides a meaningful way to fuse or merge values within universes where essentially no structure, other than an order, is assumed. Even though primarily defined over real intervals, the concept of Sugeno integral can be extended to wider domains, namely, distributive lattices, via the notion of lattice polynomial function (i.e., a combination of variables and constants using the lattice operations $\wedge$ and $\vee$ ). As it turned out, idempotent lattice polynomial functions coincide with (discrete) Sugeno integrals (see e.g. [5, 15]).

Recently, the Sugeno integral has been generalized via the notion of quasi-polynomial function (see [3]) originally defined as a mapping $f: X^{n} \rightarrow X$ on a bounded chain $X$ and which can be factorized as

$$
f\left(x_{1}, \ldots, x_{n}\right)=p\left(\varphi\left(x_{1}\right), \ldots, \varphi\left(x_{n}\right)\right),
$$

where $p: X^{n} \rightarrow X$ is a polynomial function and $\varphi: X \rightarrow X$ is an order-preserving map. This notion was later extended in two ways.

In [4, the input and output universes were allowed to be arbitrary, possibly different, bounded distributive lattices $X$ and $Y$ so that $f: X^{n} \rightarrow Y$ is factorizable as in (11), where now $p: Y^{n} \rightarrow Y$ and $\varphi: X \rightarrow Y$. These functions appear naturally within the scope of decision making under uncertainty since they subsume overall preference functionals associated with Sugeno integrals whose variables are transformed by the utility function $\varphi$. Several axiomatizations for this function class were proposed, as well as all possible factorizations described.

In 6] and 7] a different extension was considered, now appearing within the realm of multicriteria decision making. Essentially, the aggregation model was based on functions $f: X_{1} \times \cdots \times X_{n} \rightarrow Y$ for bounded chains $X_{1}, \ldots, X_{n}$ and $Y$, which can be factorized as compositions

$$
f\left(x_{1}, \ldots, x_{n}\right)=p\left(\varphi_{1}\left(x_{1}\right), \ldots, \varphi_{n}\left(x_{n}\right)\right),
$$

where $p: Y^{n} \rightarrow Y$ is a Sugeno integral, and each $\varphi_{k}: X_{k} \rightarrow Y$ is an order-preserving map. Such functions were referred to as Sugeno utility functions in 6]. Pseudo-polynomial functions were defined as functions of the form (2), where $p$ is an arbitrary (possibly non-idempotent) lattice polynomial function, and each $\varphi_{k}$ satisfies a certain boundary condition (which is weaker than order-preservation). Note that every quasi-polynomial function (11) can be regarded as a pseudo-polynomial function, where $X_{1}=\cdots=X_{n}=X$ and $\varphi_{1}=\cdots \varphi_{n}=\varphi$. Moreover, pseudo-polynomial functions naturally subsume Sugeno utility functions, and several axiomatizations were established for this function class in

Key words and phrases. Sugeno integral, distributive lattice, lattice polynomial function, pseudopolynomial function, axiomatization, factorization, multicriteria decision making. 
6. The question of factorizing a given Sugeno utility function into a composition (2) was addressed in [7, where a method for producing such a factorization was presented.

In the current paper we extend the previous results by letting $X_{1}, \ldots, X_{n}$ to be arbitrary sets and $Y$ to be an arbitrary finite distributive lattice, thus subsuming the frameworks in 4, 6, 7. Moreover, we develop general tools which allow us to produce all possible factorizations of a given pseudo-polynomial function into compositions (2) of a lattice polynomial function $p: Y^{n} \rightarrow Y$ with maps $\varphi_{k}: X_{k} \rightarrow Y$.

The structure of the paper is as follows. In Section 2 we introduce the basic notions and terminology needed throughout the paper, and recall some preliminary results. For further background on aggregation functions and their use in decision making, we refer the reader to [2, 13]; for basics in the theory of lattices, see [10, 14. In Section 3 we develop a general framework used to derive an axiomatization of pseudo-polynomial functions of somewhat different nature than those proposed in [4, 6, 7, and which will provide tools for determining all possible factorizations of given pseudo-polynomial functions in Section 4. These results are then illustrated in Section 5 by means of a concrete example, and in Section 6 we show how this new procedure can be applied to derive the algorithm provided in [7, 8].

\section{Preliminaries}

Throughout this paper, $Y$ is assumed to be a finite distributive lattice with meet and join operations denoted by $\wedge$ and $\vee$, respectively. Being finite, $Y$ has a least element and a greatest element, denoted by 0 and 1, respectively. By Birkhoff's Representation Theorem [1, $Y$ can be embedded into $\mathcal{P}(U)$, the power set of a finite set $U$. Identifying $Y$ with its image under this embedding, we will consider $Y$ as being a sublattice of $\mathcal{P}(U)$ with $0=\emptyset$ and $1=U$. The complement of a set $S \in \mathcal{P}(U)$ will be denoted by $\bar{S}$. Since $Y$ is closed under intersections, it induces a closure operator cl on $U$, and since $Y$ is closed under unions, it also induces a dual closure operator int (also known as "interior operator"):

$$
\operatorname{cl}(S):=\bigwedge_{\substack{y \in Y \\ y \geq S}} y, \quad \operatorname{int}(S):=\bigvee_{\substack{y \in Y \\ y \leq S}} y, \quad \text { for all } S \in \mathcal{P}(U) .
$$

It is easy to verify that these two operators satisfy the following identities for any $S_{1}, S_{2} \in$ $\mathcal{P}(U)$ :

$$
\operatorname{cl}\left(S_{1} \vee S_{2}\right)=\operatorname{cl}\left(S_{1}\right) \vee \operatorname{cl}\left(S_{2}\right), \quad \operatorname{int}\left(S_{1} \wedge S_{2}\right)=\operatorname{int}\left(S_{1}\right) \wedge \operatorname{int}\left(S_{2}\right) .
$$

A function $p: Y^{n} \rightarrow Y$ is a polynomial function if it can be obtained as a composition of the lattice operations $\wedge$ and $\vee$ with variables and constants. Note that polynomial functions are thus order-preserving. As observed in [15, (discrete) Sugeno integrals coincide exactly with those lattice polynomial functions $p$ which are idempotent, i.e., satisfy the identity $p(y, \ldots, y)=y$. An important lattice polynomial function (in fact, a Sugeno integral) is the median function med: $Y^{3} \rightarrow Y$ defined by

$$
\operatorname{med}\left(y_{1}, y_{2}, y_{3}\right)=\left(y_{1} \wedge y_{2}\right) \vee\left(y_{2} \wedge y_{3}\right) \vee\left(y_{3} \wedge y_{1}\right)=\left(y_{1} \vee y_{2}\right) \wedge\left(y_{2} \vee y_{3}\right) \wedge\left(y_{3} \vee y_{1}\right)
$$

It is useful to observe that the above expressions for the median can be simplified when two of the arguments are comparable:

$$
\operatorname{med}(s, y, t)=s \vee(t \wedge y) \text { whenever } s \leq t .
$$

Polynomial functions over bounded distributive lattices have very neat representations, for instance, in disjunctive normal form [12, i.e., representations by expressions of the form

$$
\bigvee_{I \subseteq[n]}\left(a_{I} \wedge \bigwedge_{i \in I} y_{i}\right)
$$

where $\bigwedge_{i \in I} y_{i}=1$ when $I=\emptyset$. To provide the "canonical" expression in this disjunctive normal form, let us define $\mathbf{1}_{I}$ to be the characteristic vector of $I \subseteq[n]:=\{1, \ldots, n\}$, i.e., the $n$-tuple in $Y^{n}$ whose $i$-th component is 1 if $i \in I$, and 0 otherwise. 
Theorem 1 (Goodstein [12]). A function $p: Y^{n} \rightarrow Y$ is a polynomial function if and only if

$$
p\left(y_{1}, \ldots, y_{n}\right)=\bigvee_{I \subseteq[n]}\left(p\left(\mathbf{1}_{I}\right) \wedge \bigwedge_{i \in I} y_{i}\right) .
$$

Remark 2. Equation (4) is referred to as the canonical disjunctive normal form representation of $p$.

Remark 3. The function given by (4) is a Sugeno integral if and only if $p(\mathbf{0})=0$ and $p(\mathbf{1})=1$.

Remark 4. Let us note that in the case $n=1$, Goodstein's theorem shows that unary polynomial functions $p$ are exactly the functions of the form $p(y)=s \vee(t \wedge y)$ with $s=p(0) \leq p(1)=t$, and these can be written as $p(y)=\operatorname{med}(s, y, t)$ according to (3).

Let $X_{1}, \ldots, X_{n}$ be arbitrary sets with at least two elements, and for each $k \in[n]$ let us fix two distinct elements $0_{X_{k}}, 1_{X_{k}}$ of $X_{k}$. We shall say that a mapping $\varphi_{k}: X_{k} \rightarrow Y$ satisfies the boundary condition (for $0_{X_{k}}$ and $1_{X_{k}}$ ) if for every $x_{k} \in X_{k}$,

$$
\varphi_{k}\left(0_{X_{k}}\right) \leq \varphi_{k}\left(x_{k}\right) \leq \varphi_{k}\left(1_{X_{k}}\right) .
$$

Observe that if $X_{k}$ is a partially ordered set with least element $0_{X_{k}}$ and greatest element $1_{X_{k}}$, and if $\varphi_{k}$ is order-preserving, then it satisfies the boundary condition (cf. also Remark 22). With no danger of ambiguity, we simply write 0 and 1 instead of $0_{X_{k}}$ and $1_{X_{k}}$ in the sequel.

A function $f: \prod_{i \in[n]} X_{i} \rightarrow Y$ is said to be a pseudo-polynomial function, if there is a polynomial function $p: Y^{n} \rightarrow Y$ and there are unary functions $\varphi_{k}: X_{k} \rightarrow Y(k \in[n])$, satisfying the boundary condition, such that

$$
f(\mathbf{x})=p(\varphi(\mathbf{x}))=p\left(\varphi_{1}\left(x_{1}\right), \ldots, \varphi_{n}\left(x_{n}\right)\right)
$$

holds for all $\mathbf{x}=\left(x_{1}, \ldots, x_{n}\right) \in \prod_{i \in[n]} X_{i}$. If $p$ is a Sugeno integral, then we say that $f$ is a pseudo-Sugeno integral. As it turns out, the notions of pseudo-polynomial function and pseudo-Sugeno integral are equivalent. This result was proved in [6, 8, for chains $Y$, but the proof given there actually just uses the fact that $Y$ is a distributive lattice and that polynomial functions are "range homogeneous", hence it applies verbatim to our setting.

Proposition 5. A function $f: \prod_{i \in[n]} X_{i} \rightarrow Y$ is a pseudo-polynomial function if and only if it is a pseudo-Sugeno integral.

Clearly, if $f$ is a pseudo-polynomial function, then it satisfies the following $n$-variable analogue of the boundary condition (5):

$$
f\left(\mathbf{x}_{k}^{0}\right) \leq f(\mathbf{x}) \leq f\left(\mathbf{x}_{k}^{1}\right) \text { for all } k \in[n], \mathbf{x} \in \prod_{i \in[n]} X_{i},
$$

where $\mathbf{x}_{k}^{a} \in \prod_{i \in[n]} X_{i}$ denotes the $n$-tuple which coincides with $\mathbf{x}$ in all but the $k$-th component, whose value is $a$.

Remark 6. Note that the particular orderings $\varphi_{k}\left(0_{X_{k}}\right) \leq \varphi_{k}\left(1_{X_{k}}\right)$ and $f\left(\mathbf{x}_{k}^{0}\right) \leq f\left(\mathbf{x}_{k}^{1}\right)$ in (5) and (7) could be reversed as the choice of $0_{X_{k}}$ and $1_{X_{k}}$ is arbitrary. Hence, the current notion of boundary condition is not more restrictive than the one used in 8 .

Next we define a property that can be used to characterize pseudo-polynomial functions. We say that $f: \prod_{i \in[n]} X_{i} \rightarrow Y$ is pseudo-median decomposable if for each $k \in[n]$ there is a unary function $\varphi_{k}: X_{k} \rightarrow Y$ satisfying (50), such that

$$
f(\mathbf{x})=\operatorname{med}\left(f\left(\mathbf{x}_{k}^{0}\right), \varphi_{k}\left(x_{k}\right), f\left(\mathbf{x}_{k}^{1}\right)\right)
$$

for every $\mathbf{x} \in \prod_{i \in[n]} X_{i}$. Note that if $f$ is pseudo-median decomposable w.r.t. unary functions $\varphi_{k}: X_{k} \rightarrow Y(k \in[n])$ satisfying (5), then (7) holds.

The following theorem shows that every pseudo-median decomposable function is a pseudo-polynomial function, and provides a disjunctive normal form of a polynomial function $p_{f}$ which can be used to factorize $f$. This theorem appears in [7, 8] for the 
special case of chains. We use the notation $\widehat{\mathbf{1}}_{I}$ for the characteristic vector of $I \subseteq[n]$ in $\prod_{i \in[n]} X_{i}$, i.e., $\widehat{\mathbf{1}}_{I} \in \prod_{i \in[n]} X_{i}$ is the $n$-tuple whose $i$-th component is $1_{X_{i}}$ if $i \in I$, and $0_{X_{i}}$ otherwise.

Theorem 7. If $f: \prod_{i \in[n]} X_{i} \rightarrow Y$ is pseudo-median decomposable w.r.t. unary functions $\varphi_{k}: X_{k} \rightarrow Y(k \in[n])$, then $f(\mathbf{x})=p_{f}(\boldsymbol{\varphi}(\mathbf{x}))$, where the polynomial function $p_{f}$ is given by

$$
p_{f}\left(y_{1}, \ldots, y_{n}\right)=\bigvee_{I \subseteq[n]}\left(f\left(\widehat{\mathbf{1}}_{I}\right) \wedge \bigwedge_{i \in I} y_{i}\right)
$$

Proof. We need to prove that the following identity holds:

$$
f\left(x_{1}, \ldots, x_{n}\right)=\bigvee_{I \subseteq[n]}\left(f\left(\widehat{\mathbf{1}}_{I}\right) \wedge \bigwedge_{i \in I} \varphi_{i}\left(x_{i}\right)\right) .
$$

We proceed by induction on $n$. If $n=1$, then the right hand side of (10) takes the form $f(0) \vee\left(f(1) \wedge \varphi_{1}\left(x_{1}\right)\right)$. From (77) it follows that $f(0) \leq f(1)$, and then, using (3), we can rewrite $f(0) \vee\left(f(1) \wedge \varphi_{1}\left(x_{1}\right)\right)$ as med $\left(f(0), \varphi_{1}\left(x_{1}\right), f(1)\right)$, which equals $f\left(x_{1}\right)$ by (8).

Now suppose that the statement of the theorem is true for all pseudo-median decomposable functions in $n-1$ variables. Let $f_{0}$ and $f_{1}$ be the $(n-1)$-ary functions defined by

$$
\begin{aligned}
& f_{0}\left(x_{1}, \ldots, x_{n-1}\right)=f\left(x_{1}, \ldots, x_{n-1}, 0\right), \\
& f_{1}\left(x_{1}, \ldots, x_{n-1}\right)=f\left(x_{1}, \ldots, x_{n-1}, 1\right) .
\end{aligned}
$$

Observe that (7) implies $f_{0} \leq f_{1}$. Applying the pseudo-median decomposition to $f$ with $k=n$ and rewriting the median using (3), we obtain

$$
\begin{aligned}
f\left(x_{1}, \ldots, x_{n}\right) & =\operatorname{med}\left(f_{0}\left(x_{1}, \ldots, x_{n-1}\right), \varphi_{n}\left(x_{n}\right), f_{1}\left(x_{1}, \ldots, x_{n-1}\right)\right) \\
& =f_{0}\left(x_{1}, \ldots, x_{n-1}\right) \vee\left(f_{1}\left(x_{1}, \ldots, x_{n-1}\right) \wedge \varphi_{n}\left(x_{n}\right)\right) .
\end{aligned}
$$

It is easy to verify that $f_{0}$ and $f_{1}$ are pseudo-median decomposable w.r.t. $\varphi_{1}, \ldots, \varphi_{n-1}$, therefore we can apply the induction hypothesis to these functions:

$$
\begin{aligned}
& f_{0}\left(x_{1}, \ldots, x_{n-1}\right)=\bigvee_{I \subseteq[n-1]}\left(f_{0}\left(\widehat{\mathbf{1}}_{I}\right) \wedge \bigwedge_{i \in I} \varphi_{i}\left(x_{i}\right)\right)=\bigvee_{I \subseteq[n-1]}\left(f\left(\widehat{\mathbf{1}}_{I}\right) \wedge \bigwedge_{i \in I} \varphi_{i}\left(x_{i}\right)\right), \\
& f_{1}\left(x_{1}, \ldots, x_{n-1}\right)=\bigvee_{I \subseteq[n-1]}\left(f_{1}\left(\widehat{\mathbf{1}}_{I}\right) \wedge \bigwedge_{i \in I} \varphi_{i}\left(x_{i}\right)\right)=\bigvee_{I \subseteq[n-1]}\left(f\left(\widehat{\mathbf{1}}_{I \cup\{n\}}\right) \wedge \bigwedge_{i \in I} \varphi_{i}\left(x_{i}\right)\right) .
\end{aligned}
$$

Substituting back into (11) and using distributivity we obtain the desired equality (10).

Now we can prove that pseudo-median decomposability actually characterizes pseudopolynomial functions (see [6, 8, for the case of chains, where the proof is slightly simpler).

Theorem 8. Let $f: \prod_{i \in[n]} X_{i} \rightarrow Y$ be a function. Then $f$ is a pseudo-polynomial function if and only if $f$ is pseudo-median decomposable.

Proof. Sufficiency follows from Theorem 7 , so we only need to show that if $f$ is a pseudopolynomial function, then it is pseudo-median decomposable. Suppose that $f(\mathbf{x})=$ $p(\boldsymbol{\varphi}(\mathbf{x}))$ as in (6), and let $k \in[n]$. We have to prove that (8) holds for all $\mathbf{x} \in \prod_{i \in[n]} X_{i}$. Regarding $x_{i}$ as a fixed element of $X_{i}$ for each $i \neq k$, we can define a unary polynomial function $u: Y \rightarrow Y$ by

$$
u(y)=p\left(\varphi_{1}\left(x_{1}\right), \ldots, \varphi_{k-1}\left(x_{k-1}\right), y, \varphi_{k+1}\left(x_{k+1}\right), \ldots, \varphi_{n}\left(x_{n}\right)\right) .
$$

To simplify notation, let us write $a:=\varphi_{k}(0), z:=\varphi_{k}\left(x_{k}\right), b:=\varphi_{k}(1)$, and let us note that the boundary condition (5) yields $a \leq z \leq b$. With this notation (8) reads as $u(z)=\operatorname{med}(u(a), z, u(b))$. In order to verify this equality, we write $u$ in disjunctive normal form as in Remark 4 $u(y)=s \vee(t \wedge y)$, where $s \leq t$. Now the proof is a straightforward computation, making heavy use of distributivity: 


$$
\begin{aligned}
\operatorname{med}(u(a), z, u(b)) & =u(a) \vee(u(b) \wedge z)=(s \vee(t \wedge a)) \vee((s \vee(t \wedge b)) \wedge z) \\
& =s \vee(t \wedge a) \vee(s \wedge z) \vee(t \wedge b \wedge z)=s \vee(t \wedge a) \vee(s \wedge z) \vee(t \wedge z) \\
& =s \vee(t \wedge a) \vee(t \wedge z)=s \vee(t \wedge(a \vee z))=s \vee(t \wedge z)=u(z)
\end{aligned}
$$

\section{Characterization of PSEudo-Polynomial Functions}

Let $f: \prod_{i \in[n]} X_{i} \rightarrow Y$ be a function satisfying (7), and for each $k \in[n]$ let us define two auxiliary functions $\Phi_{k}^{-}, \Phi_{k}^{+}: X_{k} \rightarrow Y$ as follows:

$$
\Phi_{k}^{-}\left(a_{k}\right):=\bigvee_{\mathbf{x}: x_{k}=a_{k}} \operatorname{cl}\left(f(\mathbf{x}) \wedge \overline{f\left(\mathbf{x}_{k}^{0}\right)}\right), \quad \Phi_{k}^{+}\left(a_{k}\right):=\bigwedge_{\mathbf{x}: x_{k}=a_{k}} \operatorname{int}\left(f(\mathbf{x}) \vee \overline{f\left(\mathbf{x}_{k}^{1}\right)}\right) .
$$

Here the join and the meet range over all $\mathbf{x} \in \prod_{i \in[n]} X_{i}$ whose $k$-th component is $a_{k}$. Note that from (77) it follows that $\Phi_{k}^{-}$and $\Phi_{k}^{+}$satisfy the boundary condition (5). With the help of these functions, we will give a necessary and sufficient condition for $f$ to be a pseudo-polynomial function. The following lemma formulates a simple observation that allows us to solve equation (8) for $\varphi_{k}\left(x_{k}\right)$.

Lemma 9. For any $u \leq m \leq w, v \in Y$ the following two conditions are equivalent:

(i) $\operatorname{med}(u, v, w)=m$;

(ii) $m \wedge \bar{u} \leq v \leq m \vee \bar{w}$.

Proof. Assuming that med $(u, v, w)=m$, we can estimate $m \wedge \bar{u}$ using (3) as follows:

$$
m \wedge \bar{u}=(u \vee(v \wedge w)) \wedge \bar{u}=(u \wedge \bar{u}) \vee(v \wedge w \wedge \bar{u})=0 \vee(v \wedge w \wedge \bar{u}) \leq v .
$$

An analogous argument shows that $v \leq m \vee \bar{w}$, and this establishes (i) $\Longrightarrow$ (ii)

In order to prove (ii) $\Longrightarrow$ (i) let us first compute med $(u, m \wedge \bar{u}, w)$, again with the help of (3):

$$
\operatorname{med}(u, m \wedge \bar{u}, w)=u \vee(m \wedge \bar{u} \wedge w)=(u \vee m) \wedge(u \vee \bar{u}) \wedge(u \vee w)=m \wedge 1 \wedge w=m
$$

Similarly, we have med $(u, m \vee \bar{w}, w)=m$, and then, using (ii) and the monotonicity of the median function, we conclude

$$
m=\operatorname{med}(u, m \wedge \bar{u}, w) \leq \operatorname{med}(u, v, w) \leq \operatorname{med}(u, m \vee \bar{w}, w)=m,
$$

hence $\operatorname{med}(u, v, w)=m$.

With the help of the above lemma we derive from Theorem 8 a necessary condition for $f$ to be a pseudo-polynomial function.

Proposition 10. If $f: \prod_{i \in[n]} X_{i} \rightarrow Y$ is a pseudo-polynomial function, then it satisfies (17) and

$$
\Phi_{k}^{-} \leq \Phi_{k}^{+}, \quad \text { for all } k \in[n] .
$$

Proof. Let us suppose that $f(\mathbf{x})=p(\boldsymbol{\varphi}(\mathbf{x}))$ is a pseudo-polynomial function. Then (8) holds by Theorem 8 , and applying Lemma 9 with $u=f\left(\mathbf{x}_{k}^{0}\right), m=f(\mathbf{x}), w=f\left(\mathbf{x}_{k}^{1}\right)$ and $v=\varphi_{k}\left(x_{k}\right)$, we see that $f(\mathbf{x}) \wedge \overline{f\left(\mathbf{x}_{k}^{0}\right)} \leq \varphi_{k}\left(x_{k}\right) \leq f(\mathbf{x}) \vee \overline{f\left(\mathbf{x}_{k}^{1}\right)}$. Moreover, since $\varphi_{k}\left(x_{k}\right) \in Y$, we have

$$
\operatorname{cl}\left(f(\mathbf{x}) \wedge \overline{f\left(\mathbf{x}_{k}^{0}\right)}\right) \leq \varphi_{k}\left(x_{k}\right) \leq \operatorname{int}\left(f(\mathbf{x}) \vee \overline{f\left(\mathbf{x}_{k}^{1}\right)}\right) .
$$

Considering these inequalities for all $\mathrm{x} \in \prod_{i \in[n]} X_{i}$ with a fixed $k$-th component $x_{k}=a_{k}$, it follows that

$$
\Phi_{k}^{-}\left(a_{k}\right) \leq \varphi_{k}\left(a_{k}\right) \leq \Phi_{k}^{+}\left(a_{k}\right)
$$

for all $k \in[n], a_{k} \in X_{k}$. 
Remark 11. Let us note that (13) holds if and only if each joinand in the definition of $\Phi_{k}^{-}\left(a_{k}\right)$ is less than or equal to each meetand in the definition of $\Phi_{k}^{+}\left(a_{k}\right)$. In other words, (13) is equivalent to

$$
\operatorname{cl}\left(f(\mathbf{y}) \wedge \overline{f\left(\mathbf{y}_{k}^{0}\right)}\right) \leq \operatorname{int}\left(f(\mathbf{x}) \vee \overline{f\left(\mathbf{x}_{k}^{1}\right)}\right) \text { for all } \mathbf{x}, \mathbf{y} \in \prod_{i \in[n]} X \text { with } x_{k}=y_{k} .
$$

In order to prove that the necessary condition presented in the above proposition is also sufficient, we verify that (77) and (13) imply that $f$ is pseudo-median decomposable with respect to $\Phi_{1}^{-}, \ldots, \Phi_{n}^{-}$and also with respect to $\Phi_{1}^{+}, \ldots, \Phi_{n}^{+}$.

Proposition 12. Suppose that $f: \prod_{i \in[n]} X_{i} \rightarrow Y$ satisfies (7) and (13). Then, for all $\mathbf{x} \in \prod_{i \in[n]} X_{i}$ and $k \in[n]$, we have

$$
f(\mathbf{x})=\operatorname{med}\left(f\left(\mathbf{x}_{k}^{0}\right), \Phi_{k}^{-}\left(x_{k}\right), f\left(\mathbf{x}_{k}^{1}\right)\right)=\operatorname{med}\left(f\left(\mathbf{x}_{k}^{0}\right), \Phi_{k}^{+}\left(x_{k}\right), f\left(\mathbf{x}_{k}^{1}\right)\right) .
$$

Proof. We prove that $f$ is pseudo-median decomposable with respect to $\Phi_{1}^{-}, \ldots, \Phi_{n}^{-}$, and leave to the reader the analogous argument for $\Phi_{1}^{+}, \ldots, \Phi_{n}^{+}$. Let us fix $k \in[n], a_{k} \in X_{k}$ and $\mathrm{x} \in \prod_{i \in[n]} X_{i}$ with $x_{k}=a_{k}$. By the definition of $\Phi_{k}^{-}$, we have

$$
\operatorname{med}\left(f\left(\mathbf{x}_{k}^{0}\right), \Phi_{k}^{-}\left(a_{k}\right), f\left(\mathbf{x}_{k}^{1}\right)\right)=\operatorname{med}\left(f\left(\mathbf{x}_{k}^{0}\right), \bigvee_{\mathbf{y}: y_{k}=a_{k}} \operatorname{cl}\left(f(\mathbf{y}) \wedge \overline{f\left(\mathbf{y}_{k}^{0}\right)}\right), f\left(\mathbf{x}_{k}^{1}\right)\right)
$$

From the distributivity of $Y$ it follows that joins distribute over medians, and thus:

$$
\operatorname{med}\left(f\left(\mathbf{x}_{k}^{0}\right), \Phi_{k}^{-}\left(a_{k}\right), f\left(\mathbf{x}_{k}^{1}\right)\right)=\bigvee_{\mathbf{y}: y_{k}=a_{k}} \operatorname{med}\left(f\left(\mathbf{x}_{k}^{0}\right), \operatorname{cl}\left(f(\mathbf{y}) \wedge \overline{f\left(\mathbf{y}_{k}^{0}\right)}\right), f\left(\mathbf{x}_{k}^{1}\right)\right) .
$$

We can estimate this join from below by keeping only the joinand corresponding to $\mathbf{y}=\mathbf{x}$ (this indeed appears in the join, since $\left.x_{k}=a_{k}\right)$ :

$$
\operatorname{med}\left(f\left(\mathbf{x}_{k}^{0}\right), \Phi_{k}^{-}\left(a_{k}\right), f\left(\mathbf{x}_{k}^{1}\right)\right) \geq \operatorname{med}\left(f\left(\mathbf{x}_{k}^{0}\right), \operatorname{cl}\left(f(\mathbf{x}) \wedge \overline{f\left(\mathbf{x}_{k}^{0}\right)}\right), f\left(\mathbf{x}_{k}^{1}\right)\right) .
$$

Applying Lemma 9 with $u=f\left(\mathbf{x}_{k}^{0}\right), v=\operatorname{cl}\left(f(\mathbf{x}) \wedge \overline{f\left(\mathbf{x}_{k}^{0}\right)}\right), w=f\left(\mathbf{x}_{k}^{1}\right)$ and $m=f(\mathbf{x})$ and taking into account that $f\left(\mathbf{x}_{k}^{0}\right) \leq f(\mathbf{x}) \leq f\left(\mathbf{x}_{k}^{1}\right)$ holds by (7), we see that the right hand side of (16) equals $f(\mathbf{x})$. This yields the inequality

$$
\operatorname{med}\left(f\left(\mathbf{x}_{k}^{0}\right), \Phi_{k}^{-}\left(a_{k}\right), f\left(\mathbf{x}_{k}^{1}\right)\right) \geq f(\mathbf{x}) .
$$

In order to prove the converse inequality, let us note that property (13) implies

$$
\operatorname{cl}\left(f(\mathbf{y}) \wedge \overline{f\left(\mathbf{y}_{k}^{0}\right)}\right) \leq \operatorname{int}\left(f(\mathbf{x}) \vee \overline{f\left(\mathbf{x}_{k}^{1}\right)}\right),
$$

whenever $y_{k}=a_{k}$ (see Remark 11). Thus, replacing $\operatorname{cl}\left(f(\mathbf{y}) \wedge \overline{f\left(\mathbf{y}_{k}^{0}\right)}\right)$ by $\operatorname{int}(f(\mathbf{x}) \vee$ $\left.\overline{f\left(\mathbf{x}_{k}^{1}\right)}\right)$ in each joinand on the right hand side of (15), we get the upper estimate

$$
\operatorname{med}\left(f\left(\mathbf{x}_{k}^{0}\right), \Phi_{k}^{-}\left(a_{k}\right), f\left(\mathbf{x}_{k}^{1}\right)\right) \leq \operatorname{med}\left(f\left(\mathbf{x}_{k}^{0}\right), \operatorname{int}\left(f(\mathbf{x}) \vee \overline{f\left(\mathbf{x}_{k}^{1}\right)}\right), f\left(\mathbf{x}_{k}^{1}\right)\right) .
$$

Again, Lemma 9 shows that the right hand side of (18) equals $f(\mathbf{x})$, hence we have

$$
\operatorname{med}\left(f\left(\mathbf{x}_{k}^{0}\right), \Phi_{k}^{-}\left(a_{k}\right), f\left(\mathbf{x}_{k}^{1}\right)\right) \leq f(\mathbf{x}) \text {. }
$$

Combining inequalities (17) and (19), we get the desired equality

$$
\operatorname{med}\left(f\left(\mathbf{x}_{k}^{0}\right), \Phi_{k}^{-}\left(a_{k}\right), f\left(\mathbf{x}_{k}^{1}\right)\right)=f(\mathbf{x}) .
$$

Propositions 10 and 12 together with Theorem 8 yield the following characterization of pseudo-polynomial functions.

Theorem 13. A function $f: \prod_{i \in[n]} X_{i} \rightarrow Y$ is a pseudo-polynomial function if and only if it satisfies conditions (7) and (13).

Remark 14. Theorem 13 is of different nature than Theorem 8 and the various characterizations obtained in [8]: here the necessary and sufficient condition for $f$ being a pseudo-polynomial function is given solely in terms of $f$ itself, without referring to the existence of certain functions $\varphi_{k}$. 


\section{FACTORIZATIONS OF PSEUdo-POLYNOMIAL FUnCTIONS}

Let us suppose that $f: \prod_{i \in[n]} X_{i} \rightarrow Y$ satisfies (7) and (13). According to Theorem 13, $f$ is a pseudo-polynomial function, i.e., it has a factorization of the form $f(\mathbf{x})=$ $p(\boldsymbol{\varphi}(\mathbf{x}))$, where $p: Y^{n} \rightarrow Y$ is a polynomial function and each $\varphi_{k}: X_{k} \rightarrow Y(k \in[n])$ is a unary map satisfying (15). We now show how to construct such a factorization; in fact, we will find all possible factorizations. First we describe the set of possible functions $\varphi_{k}$.

Theorem 15. For any function $f: \prod_{i \in[n]} X_{i} \rightarrow Y$ satisfying (7) and unary maps $\varphi_{k}: X_{k} \rightarrow Y(k \in[n])$ satisfying (5), the following three conditions are equivalent:

(i) $\Phi_{k}^{-} \leq \varphi_{k} \leq \Phi_{k}^{+}$holds for all $k \in[n]$;

(ii) $f(\mathbf{x})=p_{f}\left(\boldsymbol{\varphi}(\mathbf{x})\right.$ ) (where $p_{f}$ is given by (9) in Theorem $\mathbf{7 1}$ );

(iii) there exists a polynomial function $p: Y^{n} \rightarrow Y$ such that $f(\mathbf{x})=p(\varphi(\mathbf{x}))$.

Proof. The implication (ii) $\Longrightarrow$ (iii) is trivial, and (iii) $\Longrightarrow$ (i) has been established in the course of the proof of Proposition 10 (see equation (14)).

So suppose that (i) holds. Then obviously (13) holds, and Proposition 12 shows that $f$ is pseudo-median decomposable with respect to $\Phi_{1}^{-}, \ldots, \Phi_{n}^{-}$and also with respect to $\Phi_{1}^{+}, \ldots, \Phi_{n}^{+}$. Since $\Phi_{k}^{-} \leq \varphi_{k} \leq \Phi_{k}^{+}$holds for all $k \in[n]$ by (i), we have

$$
\begin{aligned}
f(\mathbf{x}) & =\operatorname{med}\left(f\left(\mathbf{x}_{k}^{0}\right), \Phi_{k}^{-}\left(x_{k}\right), f\left(\mathbf{x}_{k}^{1}\right)\right) \\
& \leq \operatorname{med}\left(f\left(\mathbf{x}_{k}^{0}\right), \varphi_{k}\left(x_{k}\right), f\left(\mathbf{x}_{k}^{1}\right)\right) \\
& \leq \operatorname{med}\left(f\left(\mathbf{x}_{k}^{0}\right), \Phi_{k}^{+}\left(x_{k}\right), f\left(\mathbf{x}_{k}^{1}\right)\right)=f(\mathbf{x}),
\end{aligned}
$$

therefore $f$ is pseudo-median decomposable with respect to $\varphi_{1}, \ldots, \varphi_{n}$. Now (ii) follows immediately from Theorem 7

Theorem 15describes all those unary maps $\varphi_{1}, \ldots, \varphi_{n}$ that can occur in a factorization of $f$, but it does not provide all possible polynomial functions $p$. (We know that $p_{f}$ can be used in any factorization, but there may be others as well.) To find all factorizations (6) of $f$, let us fix unary functions $\varphi_{k}: X_{k} \rightarrow Y(k \in[n])$ satisfying (5), such that $\Phi_{k}^{-} \leq$ $\varphi_{k} \leq \Phi_{k}^{+}$for each $k \in[n]$. To simplify notation, let $a_{k}=\varphi_{k}\left(0_{X_{k}}\right), b_{k}=\varphi_{k}\left(1_{X_{k}}\right)$, and for each $I \subseteq[n]$ let $\mathbf{e}_{I} \in Y^{n}$ be the $n$-tuple whose $i$-th component is $a_{i}$ if $i \notin I$ and $b_{i}$ if $i \in I$. If $p: Y^{n} \rightarrow Y$ is a polynomial function such that $f(\mathbf{x})=p(\boldsymbol{\varphi}(\mathbf{x}))$, then

$$
p\left(\mathbf{e}_{I}\right)=f\left(\widehat{\mathbf{1}}_{I}\right) \text { for all } I \subseteq[n],
$$

since $\mathbf{e}_{I}=\varphi\left(\widehat{\mathbf{1}}_{I}\right)$. We show that (20) is not only necessary but also sufficient to establish the factorization $f(\mathbf{x})=p(\boldsymbol{\varphi}(\mathbf{x}))$.

Lemma 16. Let $f: \prod_{i \in[n]} X_{i} \rightarrow Y$ be a function satisfying (7]) and (13), and let $\varphi_{k}: X_{k} \rightarrow Y(k \in[n])$ be maps satisfying (5), such that $\Phi_{k}^{-} \leq \varphi_{k} \leq \Phi_{k}^{+}$for all $k \in[n]$. Then a polynomial function $p: Y^{n} \rightarrow Y$ yields a factorization $f(\mathbf{x})=p(\varphi(\mathbf{x}))$ if and only if (20) holds.

Proof. As noted above, necessity is trivial. To prove the sufficiency, let us assume that $p$ satisfies (20), and let us define a function $f^{\prime}: \prod_{i \in[n]} X_{i} \rightarrow Y$ by $f^{\prime}(\mathbf{x})=p(\boldsymbol{\varphi}(\mathbf{x}))$. Then $f^{\prime}$ is a pseudo-polynomial function, and by Theorem 8 it is pseudo-median decomposable with respect to $\varphi_{1}, \ldots, \varphi_{n}$. From Theorem 7 we get the following expression for $f^{\prime}$ :

$$
f^{\prime}(\mathbf{x})=\bigvee_{I \subseteq[n]}\left(f^{\prime}\left(\widehat{\mathbf{1}}_{I}\right) \wedge \bigwedge_{i \in I} \varphi_{i}\left(x_{i}\right)\right) .
$$

The assumptions on $f$ and $\varphi_{k}$ guarantee that $f(\mathbf{x})=p_{f}(\boldsymbol{\varphi}(\mathbf{x}))$ by Theorem 15, and hence $f$ can be written as

$$
f(\mathbf{x})=\bigvee_{I \subseteq[n]}\left(f\left(\widehat{\mathbf{1}}_{I}\right) \wedge \bigwedge_{i \in I} \varphi_{i}\left(x_{i}\right)\right) .
$$

Since $f^{\prime}\left(\widehat{\mathbf{1}}_{I}\right)=p\left(\boldsymbol{\varphi}\left(\widehat{\mathbf{1}}_{I}\right)\right)=p\left(\mathbf{e}_{I}\right)$ and $p\left(\mathbf{e}_{I}\right)=f\left(\widehat{\mathbf{1}}_{I}\right)$ by (20), it follows from (21) and (22) that $f^{\prime}(\mathbf{x})=f(\mathbf{x})$. 
TABLE 1. The airline example

(a) The function $f$

\begin{tabular}{cc|c}
$x_{1}$ & $x_{2}$ & $f\left(x_{1}, x_{2}\right)$ \\
\hline $\mathrm{A}_{1}$ & $\mathrm{E}$ & $\mathrm{B}$ \\
$\mathrm{A}_{1}$ & $\mathrm{~F}$ & $\mathrm{~B}$ \\
$\mathrm{~A}_{2}$ & $\mathrm{E}$ & $\mathrm{B}$ \\
$\mathrm{A}_{2}$ & $\mathrm{~F}$ & $\mathrm{D}$ \\
$\mathrm{A}_{3}$ & $\mathrm{E}$ & $\mathrm{N}$ \\
$\mathrm{A}_{3}$ & $\mathrm{~F}$ & $\mathrm{G}$ \\
$\mathrm{A}_{4}$ & $\mathrm{E}$ & $\mathrm{N}$ \\
$\mathrm{A}_{4}$ & $\mathrm{~F}$ & $\mathrm{~V}$
\end{tabular}

(b) The functions $\Phi_{k}^{-}, \Phi_{k}^{+}$

\begin{tabular}{c|cc}
$x_{1}$ & $\Phi_{1}^{-}\left(x_{1}\right)$ & $\Phi_{1}^{+}\left(x_{1}\right)$ \\
\hline $\mathrm{A}_{1}$ & $\mathrm{~B}$ & $\mathrm{~B}$ \\
$\mathrm{~A}_{2}$ & $\mathrm{D}$ & $\mathrm{D}$ \\
$\mathrm{A}_{3}$ & $\mathrm{G}$ & $\mathrm{G}$ \\
$\mathrm{A}_{4}$ & $\mathrm{~V}$ & $\mathrm{~V}$
\end{tabular}

\begin{tabular}{l|cc}
$x_{2}$ & $\Phi_{2}^{-}\left(x_{2}\right)$ & $\Phi_{2}^{+}\left(x_{2}\right)$ \\
\hline $\mathrm{E}$ & $\mathrm{B}$ & $\mathrm{N}$ \\
$\mathrm{F}$ & $\mathrm{V}$ & $\mathrm{V}$
\end{tabular}

For a given $f$ and given $a_{k}, b_{k} \in Y$, (20) gives rise to a polynomial interpolation problem over $Y$ : the values of the unknown polynomial function $p$ are prescribed at certain $\left(2^{n}\right.$ many) points in $Y^{n}$. It has been shown in [9] that the least solution of this interpolation problem is

$$
p^{-}(\mathbf{y})=\bigvee_{I \subseteq[n]}\left(c_{I}^{-} \wedge \bigwedge_{i \in I} y_{i}\right), \quad \text { where } c_{I}^{-}=\operatorname{cl}\left(f\left(\widehat{\mathbf{1}}_{I}\right) \wedge \bigwedge_{i \notin I} \overline{a_{i}}\right),
$$

whereas the greatest solution is

$$
p^{+}(\mathbf{y})=\bigvee_{I \subseteq[n]}\left(c_{I}^{+} \wedge \bigwedge_{i \in I} y_{i}\right), \quad \text { where } c_{I}^{+}=\operatorname{int}\left(f\left(\widehat{\mathbf{1}}_{I}\right) \vee \bigvee_{i \in I} \overline{b_{i}}\right) .
$$

In other words, a polynomial function $p$ is a solution of (20) if and only if $p^{-} \leq p \leq p^{+}$. Since, by Theorem $1, p$ is uniquely determined by its values on the tuples $\mathbf{1}_{I}(I \subseteq[n])$, this is equivalent to

$$
c_{I}^{-}=p^{-}\left(\mathbf{1}_{I}\right) \leq p\left(\mathbf{1}_{I}\right) \leq p^{+}\left(\mathbf{1}_{I}\right)=c_{I}^{+} \text {for all } I \subseteq[n] .
$$

Thus we obtain the following description of all possible factorizations of a given pseudopolynomial function $f$.

Theorem 17. Let $f: \prod_{i \in[n]} X_{i} \rightarrow Y$ be a function satisfying (77), for each $k \in[n]$ let $\varphi_{k}: X_{k} \rightarrow Y$ be a given function satisfying (5), and let $p: Y^{n} \rightarrow Y$ be a polynomial function. Then $f(\mathbf{x})=p(\boldsymbol{\varphi}(\mathbf{x}))$ if and only if $\Phi_{k}^{-} \leq \varphi_{k} \leq \Phi_{k}^{+}$for each $k \in[n]$, and we have $p^{-} \leq p \leq p^{+}$.

Remark 18. Note that the polynomial functions $p^{-}$and $p^{+}$are defined in terms of the maps $\varphi_{k}$, hence we have to choose these maps first, and only then we can determine $p^{-}$ and $p^{+}$(cf. the example in Section [5).

Remark 19. Clearly, $c_{I}^{-} \leq f\left(\widehat{\mathbf{1}}_{I}\right) \leq c_{I}^{+}$holds independently of $a_{k}, b_{k}$, hence the polynomial function $p_{f}$ can be used in any factorization of $f$, as it was already shown in Theorem 7

Remark 20. If $X_{k}$ is a partially ordered set for each $k \in[n]$ and $f$ is order-preserving, then $\Phi_{k}^{-}$and $\Phi_{k}^{+}$are also order-preserving. This shows that every order-preserving pseudo-polynomial function has a factorization where each $\varphi_{k}$ is order-preserving. Consequently, order-preserving pseudo-Sugeno integrals coincide with Sugeno utility functions (cf. Corollary 2 in 8 ).

\section{An eXAmple}

We illustrate the results of the previous section with a simple example, where preferences about travelling with four airlines $\mathrm{A}_{1}, \mathrm{~A}_{2}, \mathrm{~A}_{3}, \mathrm{~A}_{4}$ in economy class (E) and first class (F) are modelled by pseudo-polynomial functions. Table 1(a) shows a fictitious customer's evaluation of these eight options, where B,N, G, V stand for "bad", "neutral", 

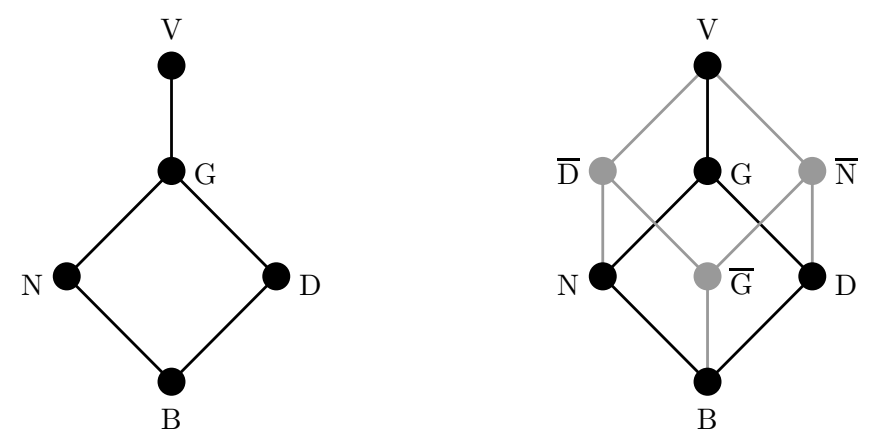

Figure 1. The lattice $Y$ and its embedding into a power set

"good" and "very good", respectively, and D means "don't know". This table defines an overall preference function $f: X_{1} \times X_{2} \rightarrow Y$, where

$$
X_{1}:=\left\{\mathrm{A}_{1}, \mathrm{~A}_{2}, \mathrm{~A}_{3}, \mathrm{~A}_{4}\right\}, X_{2}:=\{\mathrm{E}, \mathrm{F}\}, Y:=\{\mathrm{B}, \mathrm{N}, \mathrm{G}, \mathrm{V}, \mathrm{D}\} .
$$

It is plausible that $\mathrm{D}$ is better than $\mathrm{B}$, worse than $\mathrm{G}$, and incomparable with $\mathrm{N}$, hence the ordering of $Y$ is the one given in Figure 1. This is a distributive lattice that can be embedded into the power set of a three-element set $U$ as shown in Figure 1 . The figure does not indicate the representation of each element of $Y$ as a subset of $U$, only the complements of the elements, since this is all we need in order to perform the computations that follow. (Note that B and V are the complements of each other.)

For $y \in Y$ we have obviously $\operatorname{cl}(y)=\operatorname{int}(y)=y$, and for the three "extra" elements the closures and interiors can be read easily from Figure 1

$$
\begin{aligned}
\operatorname{cl}(\overline{\mathrm{D}}) & =\mathrm{V}, \quad \operatorname{cl}(\overline{\mathrm{N}})=\mathrm{V}, \quad \operatorname{cl}(\overline{\mathrm{G}})=\mathrm{V}, \\
\operatorname{int}(\overline{\mathrm{D}}) & =\mathrm{N}, \quad \operatorname{int}(\overline{\mathrm{N}})=\mathrm{D}, \quad \operatorname{int}(\overline{\mathrm{G}})=\mathrm{B} .
\end{aligned}
$$

It is obvious that $0_{X_{2}}=\mathrm{E}$ and $1_{X_{2}}=\mathrm{F}$, but $0_{X_{1}}$ and $1_{X_{1}}$ are not clear. However, from Table 1(a) we can infer that $0_{X_{1}}=A_{1}$ and $1_{X_{1}}=A_{4}$ if $f$ satisfies (7) at all. (If not, then $f$ is not a pseudo-polynomial function.)

Table 1(b) shows the auxiliary functions $\Phi_{k}^{-}, \Phi_{k}^{+}$corresponding to the function $f$. We give the details of the computation of $\Phi_{2}^{+}(\mathrm{E})$, the other values can be calculated similarly:

$$
\begin{aligned}
\Phi_{2}^{+}(\mathrm{E}) & =\bigwedge_{x_{1} \in X_{1}} \operatorname{int}\left(f\left(x_{1}, \mathrm{E}\right) \vee \overline{f\left(x_{1}, \mathrm{~F}\right)}\right) \\
& =\operatorname{int}(\mathrm{B} \vee \overline{\mathrm{B}}) \wedge \operatorname{int}(\mathrm{B} \vee \overline{\mathrm{D}}) \wedge \operatorname{int}(\mathrm{N} \vee \overline{\mathrm{G}}) \wedge \operatorname{int}(\mathrm{N} \vee \overline{\mathrm{V}}) \\
& =\operatorname{int}(\mathrm{V}) \wedge \operatorname{int}(\overline{\mathrm{D}}) \wedge \operatorname{int}(\overline{\mathrm{D}}) \wedge \operatorname{int}(\mathrm{N})=\mathrm{V} \wedge \mathrm{N} \wedge \mathrm{N} \wedge \mathrm{N}=\mathrm{N}
\end{aligned}
$$

We can see that $\Phi_{k}^{-} \leq \Phi_{k}^{+}$for $k=1,2$, therefore $f$ is a pseudo-polynomial function by Theorem 13. Theorem[15implies that in any factorization $f\left(x_{1}, x_{2}\right)=p\left(\varphi_{1}\left(x_{1}\right), \varphi_{2}\left(x_{2}\right)\right)$ of $f$, we must have $\varphi_{1}=\Phi_{1}^{-}=\Phi_{1}^{+}$, while we have two possibilities for $\varphi_{2}$ (as $\varphi_{2}(\mathrm{E})$ can be chosen to be B or $\mathrm{N}$, and $\varphi_{2}(\mathrm{~F})$ must be $\left.\mathrm{V}\right)$. Thus there are two pairs of functions $\left(\varphi_{1}, \varphi_{2}\right)$, namely $\left(\Phi_{1}^{-}, \Phi_{2}^{-}\right)$and $\left(\Phi_{1}^{+}, \Phi_{2}^{+}\right)$that allow us to factorize $f$. Theorem 15 also shows that in both cases one can use the polynomial function

$p_{f}\left(y_{1}, y_{2}\right)=\mathrm{B} \vee\left(y_{1} \wedge \mathrm{N}\right) \vee\left(y_{2} \wedge \mathrm{B}\right) \vee\left(\mathrm{V} \wedge y_{1} \wedge y_{2}\right)=\left(y_{1} \wedge \mathrm{N}\right) \vee\left(y_{1} \wedge y_{2}\right)=y_{1} \wedge\left(y_{2} \vee \mathrm{N}\right)$.

Computing the coefficients $c_{I}^{-}, c_{I}^{+}$for $\left(\Phi_{1}^{-}, \Phi_{2}^{-}\right)$, one can see that in this case $p^{-}=p_{f}=p^{+}$, i.e., $p=p_{f}$ is the only polynomial function such that $f\left(x_{1}, x_{2}\right)=$ $p\left(\Phi_{1}^{-}\left(x_{1}\right), \Phi_{2}^{-}\left(x_{2}\right)\right)$. On the other hand, choosing $\left(\varphi_{1}, \varphi_{2}\right)=\left(\Phi_{1}^{+}, \Phi_{2}^{+}\right)$, we obtain $p^{-}=y_{1} \wedge y_{2}$ and $p^{+}=p_{f}$, and these are the only possibilities, since there is no polynomial function strictly between $p^{-}$and $p^{+}$. Thus $f$ has altogether three factorizations:

$$
f\left(x_{1}, x_{2}\right)=\Phi_{1}^{-}\left(x_{1}\right) \wedge\left(\Phi_{2}^{-}\left(x_{2}\right) \vee \mathrm{N}\right)=\Phi_{1}^{+}\left(x_{1}\right) \wedge\left(\Phi_{2}^{+}\left(x_{2}\right) \vee \mathrm{N}\right)=\Phi_{1}^{+}\left(x_{1}\right) \wedge \Phi_{2}^{+}\left(x_{2}\right) .
$$

Note that these factorizations are essentially the same, since $\Phi_{2}^{-} \vee \mathrm{N}=\Phi_{2}^{+} \vee \mathrm{N}=\Phi_{2}^{+}$. The meaning of $\Phi_{2}^{+}$is pretty obvious, and $\Phi_{1}^{+}$shows the customer's opinion about the 


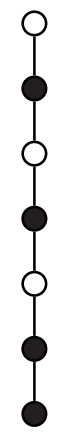

$S$

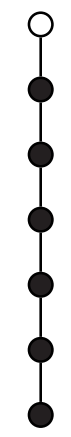

$\operatorname{cl}(S)$

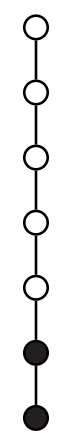

$\operatorname{int}(S)$

FiguRE 2. The closure and interior of a subset of a chain

four airlines, either based on past experience or on information received from other sources (except for airline $\mathrm{A}_{2}$, where the value $\mathrm{D}$ indicates the lack of information). The fact that $\Phi_{1}^{+}\left(x_{1}\right)$ and $\Phi_{2}^{+}\left(x_{2}\right)$ are aggregated in a conjunctive manner indicates a pessimistic attitude: the customer expects an enjoyable flight only if both the airline and the travel class are good enough.

\section{Pseudo-Polynomial FunCtions OVER Chains}

In this section we consider the case when $Y$ is a finite chain. As we will see, in this case the results of Section 4 lead to a generalization of Algorithm SUFF presented in 8. As before, we will suppose that $Y$ is a sublattice of $\mathcal{P}(U)$ for some finite set $U$, with least element $\emptyset$ and greatest element $U$. We may assume without loss of generality that $U=[m]=\{1,2, \ldots, m\}$, and $Y=\{[0],[1], \ldots,[m]\}$, where $[0]=\emptyset$. The closure of a set $S \subseteq U$ is the smallest set of the form $[k]$ that contains $S$, while the interior of $S$ is the largest set of the form $[k]$ that is contained in $S$ (see Figure 2). Formally, we have

$$
\operatorname{cl}(S)=[\max S], \quad \operatorname{int}(S)=[\min \bar{S}-1] .
$$

Let us assume that $f: \prod_{i \in[n]} X_{i} \rightarrow Y$ satisfies (7). Then $f\left(\mathbf{x}_{k}^{0}\right)=[u], f(\mathbf{x})=$ $[v], f\left(\mathbf{x}_{k}^{1}\right)=[w]$ with $u \leq v \leq w$, hence we have

$$
\begin{aligned}
& f(\mathbf{x}) \wedge \overline{f\left(\mathbf{x}_{k}^{0}\right)}=\{u+1, \ldots, v\}, \\
& f(\mathbf{x}) \vee \overline{f\left(\mathbf{x}_{k}^{1}\right)}=\{1, \ldots, v, w+1, \ldots, m\} .
\end{aligned}
$$

Therefore the terms in the definition of $\Phi_{k}^{-}$and $\Phi_{k}^{+}$can be determined as follows:

$$
\begin{array}{r}
\operatorname{cl}\left(f(\mathbf{x}) \wedge \overline{f\left(\mathbf{x}_{k}^{0}\right)}\right)= \begin{cases}f(\mathbf{x}), & \text { if } f\left(\mathbf{x}_{k}^{0}\right)<f(\mathbf{x}) ; \\
\emptyset, & \text { if } f\left(\mathbf{x}_{k}^{0}\right)=f(\mathbf{x}) ;\end{cases} \\
\operatorname{int}\left(f(\mathbf{x}) \vee \overline{f\left(\mathbf{x}_{k}^{1}\right)}\right)= \begin{cases}f(\mathbf{x}), & \text { if } f\left(\mathbf{x}_{k}^{1}\right)>f(\mathbf{x}) ; \\
U, & \text { if } f\left(\mathbf{x}_{k}^{1}\right)=f(\mathbf{x}) .\end{cases}
\end{array}
$$

Thus we obtain from Theorem [13 and Remark 11 the following characterization of pseudo-polynomial functions valued in a chain.

Theorem 21. If $Y$ is a finite chain, then a function $f: \prod_{i \in[n]} X_{i} \rightarrow Y$ is a pseudopolynomial function if and only if it satisfies condition (77) and

$$
f\left(\mathbf{x}_{k}^{0}\right)<f\left(\mathbf{x}_{k}^{a_{k}}\right) \text { and } f\left(\mathbf{y}_{k}^{a_{k}}\right)<f\left(\mathbf{y}_{k}^{1}\right) \Longrightarrow f\left(\mathbf{x}_{k}^{a_{k}}\right) \leq f\left(\mathbf{y}_{k}^{a_{k}}\right)
$$

holds for all $\mathbf{x}, \mathbf{y} \in \prod_{i \in[n]} X_{i}$ and $k \in[n], a_{k} \in X_{k}$.

Let us now define the following three sets for any $k \in[n], a_{k} \in X_{k}$, as in [8]:

$$
\begin{aligned}
\mathcal{W}_{a_{k}}^{f} & =\left\{f(\mathbf{x}): x_{k}=a_{k} \text { and } f\left(\mathbf{x}_{k}^{0}\right)<f(\mathbf{x})<f\left(\mathbf{x}_{k}^{1}\right)\right\}, \\
\mathcal{L}_{a_{k}}^{f} & =\left\{f(\mathbf{x}): x_{k}=a_{k} \text { and } f\left(\mathbf{x}_{k}^{0}\right)<f(\mathbf{x})=f\left(\mathbf{x}_{k}^{1}\right)\right\}, \\
\mathcal{U}_{a_{k}}^{f} & =\left\{f(\mathbf{x}): x_{k}=a_{k} \text { and } f\left(\mathbf{x}_{k}^{0}\right)=f(\mathbf{x})<f\left(\mathbf{x}_{k}^{1}\right)\right\} .
\end{aligned}
$$


From (23) and (24) it follows that $\Phi_{k}^{-}\left(a_{k}\right)=\bigvee \mathcal{L}_{a_{k}}^{f} \vee \bigvee \mathcal{W}_{a_{k}}^{f}$ and $\Phi_{k}^{+}\left(a_{k}\right)=\wedge \mathcal{U}_{a_{k}}^{f} \wedge$ $\wedge \mathcal{W}_{a_{k}}^{f}$, hence the condition $\Phi_{k}^{-} \leq \varphi_{k} \leq \Phi_{k}^{+}$in Theorem 15 can be reformulated as follows:

(a) either $\mathcal{W}_{a_{k}}^{f}=\left\{\varphi_{k}\left(a_{k}\right)\right\}$ or $\mathcal{W}_{a_{k}}^{f}=\emptyset$;

(b) $\varphi_{k}\left(a_{k}\right) \geq \bigvee \mathcal{L}_{a_{k}}^{f}$;

(c) $\varphi_{k}\left(a_{k}\right) \leq \wedge \mathcal{U}_{a_{k}}^{f}$.

Thus by Theorem 15, $f$ is a pseudo-polynomial function if and only if there are functions $\varphi_{k}$ satisfying the above three conditions. If each $X_{k}$ is a bounded chain and $f$ is an order-preserving function depending on all of its variables, then $(\mathrm{a})[(\mathrm{b}) \mid(\mathrm{c})]$ are equivalent to equation (18) in [8], and Algorithm SUFF does not return the value false if and only if (13) holds. Thus, in the finite case, Theorem 7 of [8] follows as a special case of Theorem [15. Moreover, the results of Section 4 not only generalize Algorithm SUFF to arbitrary finite distributive lattices (instead of finite chains) and to pseudo-polynomial functions (instead of Sugeno utility functions), but they provide all possible factorizations of a given pseudo-polynomial function $f$ (whereas Algorithm SUFF constructs only one factorization).

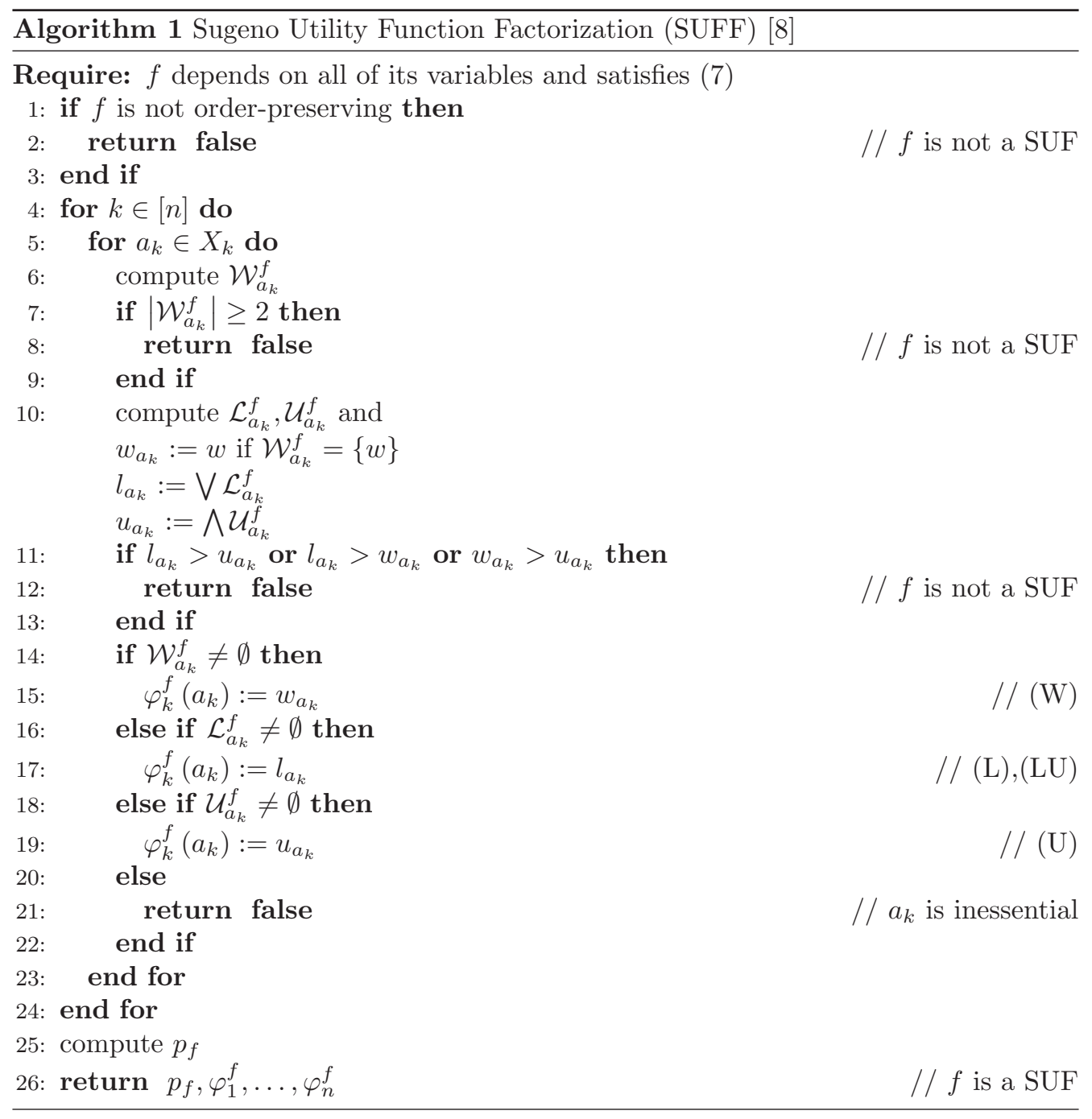

Remark 22. If the lattice $Y$ is a finite chain, as it is the case in many applications, then any map $\varphi_{k}: X_{k} \rightarrow Y$ attains its minimum and its maximum at some points in $X_{k}$, hence there exist elements $0_{X_{k}}$ and $1_{X_{k}}$ such that (5) holds. Thus, the boundary condition does not impose any restriction on $\varphi_{k}$; the point is that we must know the 
elements $0_{X_{k}}$ and $1_{X_{k}}$, even if we do not know the function $\varphi_{k}$. However, even this mild assumption can be released, by suitably modifying the definition of the functions $\Phi_{k}^{-}$ and $\Phi_{k}^{+}$, so that they do not refer to 0 and 1 anymore:

$$
\Phi_{k}^{-}\left(a_{k}\right):=\bigvee_{\substack{\mathbf{x}: x_{k}=a_{k} \\ \mathcal{\rho} \in X_{k}}} \operatorname{cl}\left(f(\mathbf{x}) \wedge \overline{f\left(\mathbf{x}_{k}^{\mathcal{\rho}}\right)}\right), \quad \Phi_{k}^{+}\left(a_{k}\right):=\bigwedge_{\substack{\mathbf{x}: x_{k}=a_{k} \\ \mathcal{\rho} \in X_{k}}} \operatorname{int}\left(f(\mathbf{x}) \vee \overline{f\left(\mathbf{x}_{k}^{\mathcal{D}}\right)}\right) .
$$

To see that these new definitions yield the same functions as (12), we only need to observe that we have just added some new joinands to the join defining $\Phi_{k}^{-}$, but each of these new joinands is dominated by some of the original joinands of (12). Indeed, from (77) it follows that $\overline{f\left(\mathbf{x}_{k}^{\mathcal{D}}\right)} \leq \overline{f\left(\mathbf{x}_{k}^{0}\right)}$ for all $\varnothing \in X_{k}$, hence

$$
\operatorname{cl}\left(f(\mathbf{x}) \wedge \overline{f\left(\mathbf{x}_{k}^{\mathcal{\rho}}\right)}\right) \leq \operatorname{cl}\left(f(\mathbf{x}) \wedge \overline{f\left(\mathbf{x}_{k}^{0}\right)}\right) .
$$

Similarly, each of the new meetands that we have added to the meet defining $\Phi_{k}^{+}$is absorbed by some of the original meetands.

With these new definitions, we can apply Theorem 13 to decide whether a given function $f$ is a pseudo-polynomial function, and we can find all maps $\varphi_{k}$ that can appear in a factorization of $f$ with the help of Theorem 15. Once we have the maps $\varphi_{k}$, we can define $0_{X_{k}}$ and $1_{X_{k}}$ as

$$
0_{X_{k}}:=\underset{x_{k} \in X_{k}}{\operatorname{argmin}} \varphi_{k}\left(x_{k}\right) \text { and } 1_{X_{k}}:=\underset{x_{k} \in X_{k}}{\operatorname{argmax}} \varphi_{k}\left(x_{k}\right),
$$

and then we can use Theorem 17 to find the possible polynomial functions $p$. (Here argmin and argmax denote the elements of $X_{k}$ where $\varphi_{k}$ attains its minimum and its maximum, respectively.) The price that we have to pay for this generality is that the computation of $\Phi_{k}^{-}$and $\Phi_{k}^{+}$is longer. Alternatively, we can apply "reverse engineering" to (7), as in Section 5, in order to find $0_{X_{k}}$ and $1_{X_{k}}$.

\section{CONCLUding REMARKS}

We have extended the study of Sugeno utility functions over chains developed in 6. 17, 8, to the case of finite distributive lattices. We refined the axiomatization given in [6, 8] by providing necessary and sufficient conditions for a function defined on a Cartesian product of arbitrary underlying sets and valued in a finite distributive lattice, to be factorizable as a pseudo-polynomial function (6). Moreover, in doing so, we were able to furnish all possible factorizations, if such a factorization exists, and we proposed a new procedure for constructing them, which subsumes that of [7, 8] in the case when the codomain is a finite chain.

Looking at directions for further research, we are inevitably drawn to the two following topics. As mentioned, pseudo-polynomial functions play an important role in multicriteria decision making since they subsume the so-called Sugeno utility functions, which in turn are used to model preference relations: Say that a preference relation $\preceq$ on $X_{1} \times \cdots \times X_{n}$ is Sugeno representable if there is a Sugeno utility function $U: X_{1} \times \cdots \times X_{n} \rightarrow Y$ such that $\mathbf{x} \preceq \mathbf{y}$ if and only if $U(\mathbf{x}) \leq U(\mathbf{y})$. Given the results of the current paper, and following the line of research developed in [2, 11, 16, it is natural to consider the following problem.

Problem 23. Axiomatize those preference relations that are Sugeno representable.

This problem was solved in the realm of decision making under uncertainty in [11.

The second emerging topic is of somewhat different nature. So far, we have played within the setting where no information is missing. However, in real-life situations this is rarely the case. Translating it into mathematical terms, it is often the case that information about the functions we deal with is incomplete. In other words, we are given partial functions in the sense that they are not everywhere defined. Taking the simplest case where the only aggregation functions considered are Sugeno integrals, we are faced with the following interpolation problem. 
Problem 24. Given a partial function $f: D \rightarrow X$, where $X$ is a distributive lattice and $D \subseteq X^{n}$, give necessary and sufficient conditions for the existence of a Sugeno integral $p: X^{n} \rightarrow X$ which interpolates $f$ on all of its domain $D$, i.e., $\left.p\right|_{D}=f$. If such a Sugeno integral exists, provide a procedure to compute it.

Again, this problem has been solved for the case of finite chains $X$ in 16 .

These two problems constitute topics of current research being carried out by the authors.

Acknowledgments. The first named author is supported by the internal research project F1R-MTH-PUL-09MRDO of the University of Luxembourg. The second named author acknowledges that the present project is supported by the TÁMOP-4.2.1/B-09/1/KONV2010-0005 program of National Development Agency of Hungary, by the Hungarian National Foundation for Scientific Research under grants no. K77409 and K83219, by the National Research Fund of Luxembourg, and cofunded under the Marie Curie Actions of the European Commission (FP7-COFUND).

\section{REFERENCES}

[1] G. Birkhoff. On the combination of subalgebras. Proc. Camb. Phil. Soc., 29 (1933) 441-464.

[2] D. Bouyssou, D. Dubois, H. Prade, M. Pirlot (eds) Decision-Making Process - Concepts and Methods, ISTE/John Wiley, 2009.

[3] M. Couceiro, J.-L. Marichal. Axiomatizations of quasi-polynomial functions on bounded chains. Aeq. Math., 396(1) (2009) 195-213.

[4] M. Couceiro, J.-L. Marichal. Axiomatizations of quasi-polynomial functions over bounded distributive lattices. Aeq. Math., 80(3) (2010) 319-334.

[5] M. Couceiro, J.-L. Marichal. Characterizations of discrete Sugeno integrals as polynomial functions over distributive lattices. Fuzzy Sets and Systems, 161(5) (2010) 694-707.

[6] M. Couceiro, T. Waldhauser, Sugeno utility functions I: Axiomatizations, in: Torra, V., Narukawa, Y., Daumas, M. (eds.), MDAI 2010, LNCS (LNAI), vol. 6408, Springer, Heidelberg, 2010, pp. 79-90.

[7] M. Couceiro, T. Waldhauser, Sugeno utility functions II: Factorizations, in: Torra, V., Narukawa, Y., Daumas, M. (eds.), MDAI 2010, LNCS (LNAI), vol. 6408, Springer, Heidelberg, 2010, pp. $91-103$.

[8] M. Couceiro, T. Waldhauser, Axiomatizations and factorizations of Sugeno utility functions, Internat. J. Uncertain. Fuzziness Knowledge-Based Systems, 19(4) (2011) 635-658.

[9] M. Couceiro, T. Waldhauser, Interpolation by polynomial functions of distributive lattices: a generalization of a theorem of R. L. Goodstein. To appear in Algebra Universalis. http://arxiv.org/abs/1110.0321

[10] B. A. Davey and H. A. Priestley. Introduction to Lattices and Order. Cambridge University Press, New York, second ed., 2002.

[11] D. Dubois, H. Prade, R. Sabbadin, Qualitative decision theory with Sugeno integrals, Fuzzy measures and integrals, Stud. Fuzziness Soft Comput., vol. 40, Physica, Heidelberg, 2000, pp. 314-332.

[12] R. L. Goodstein, The solution of equations in a lattice. Proc. Roy. Soc. Edinburgh Sect. A, 67 (1965/1967) 231-242.

[13] M. Grabisch, J.-L. Marichal, R. Mesiar, E. Pap, Aggregation Functions. Encyclopedia of Mathematics and its Applications. Cambridge University Press, Cambridge, UK, 2009.

[14] G. Grätzer, General Lattice Theory, second ed., Birkhäuser Verlag, Berlin, 2003.

[15] J.-L. Marichal, Weighted lattice polynomials, Discrete Math., 309(4) (2009) 814-820.

[16] A. Rico, M. Grabisch, Ch. Labreuche, A. Chateauneuf, Preference modeling on totally ordered sets by the Sugeno integral, Discrete Appl. Math., 147(1) (2005) 113-124.

[17] M. Sugeno. Theory of fuzzy integrals and its applications. PhD thesis, Tokyo Institute of Technology, Tokyo, 1974.

[18] M. Sugeno. Fuzzy measures and fuzzy integrals - a survey, in: M. M. Gupta, G. N. Saridis, B. R. Gaines (eds.), Fuzzy automata and decision processes, North-Holland, New York, 1977, pp. 89-102.

(M. Couceiro) Mathematics Research Unit, FStC, University of Luxembourg, 6, Rue CoudenhoveKalergi, L-1359 Luxembourg, Luxembourg and Lamsade-University Paris Dauphine, Place du Maréchal de Lattre de Tassigny, 75775 Paris Cedex 16, France

E-mail address: miguel.couceiro@uni.lu

(T. Waldhauser) Mathematics Research Unit, FSTC, University of Luxembourg, 6, Rue Coudenhove-Kalergi, L-1359 Luxembourg, Luxembourg, and Bolyai Institute, University of Szeged, Aradi vértanúk tere 1, H-6720 Szeged, Hungary

E-mail address: twaldha@math.u-szeged.hu 